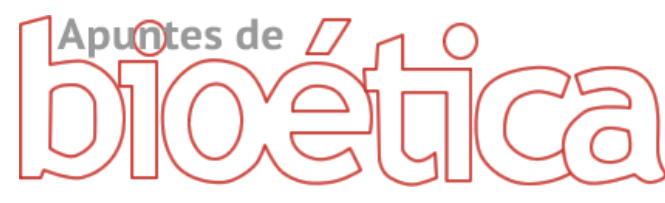

https: //doi.org/10.35383/apuntes.v4i2.679 e-ISSN:2663-4910

Universidad Católica Santo Toribio de Mogrovejo

\title{
Mujer y bioética. Experiencias y sugerencias educativas
}

\section{Woman and bioethics. Experiences and educative suggestions}

\author{
Dalia Jaqueline Santa Cruz-Vera ${ }^{1 \text {, a }}$ \\ djsantacruz@ucatolica.edu.co \\ https://orcid.org/0000-0001-7365-154X
}

Marisol Bolívar-Ramírez ${ }^{*}$, b

mbolivar@ucatolica.edu.co

https://orcid.org/0000-0002-1298-7442

\section{Laura Isabel Tobón-Gallego ${ }^{2, c}$}

lauraisabel.tobon@upb.edu.co

https://orcid.org/0000-0001-9429-5767

\section{Sagrario Ortiz-Núñez ${ }^{2, d}$}

sagrario.ortiz@upb.edu.co

https://orcid.org/0000-0002-7051-5128

\author{
*Autor corresponsal \\ 1 Universidad Católica de Colombia, Bogotá, \\ Colombia \\ 2 Universidad Pontificia Bolivariana, Medellín, \\ Colombia \\ ${ }^{\mathrm{a}}$ Master en Bioetica (Città Del Vaticano)

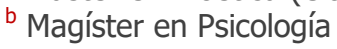 \\ c Magister en Asesoría Familiar y Gestión de \\ Programas para la Familia \\ d Doctorado Programa Periferias, Sostenibilidad y \\ Vitalidad Urbana
}

\section{Fechas importantes}

Recibido: 09/10/2021

Aceptado: $21 / 12 / 2021$

Publicado online: 23/12/2021

\section{Resumen}

El urgente reclamo de una presencia más incisiva de la mujer en la vida política, social y cultural se realiza desde diferentes frentes, y con muy diversas motivaciones. Sin embargo, el aporte genuino que a ella le corresponde, podría frustrarse cuando se descuidan las características constitutivas de la mujer. Ante este riego, el ámbito educativo se presenta como una palestra idónea para conocer la esencia femenina y al mismo tiempo impregnar con ella la práctica docente. El presente artículo es fruto de la investigación y enseñanza de la bioética inserta en el currículo de Humanidades y Enfermería, y tiene por objetivo profundizar en las cualidades de lo auténtico femenino para mostrar en qué medida podrían enriquecer la praxis educativa universitaria. Los referentes teóricos son, principalmente, las reflexiones antropológicas sobre la mujer de Edith Stein y Juan Pablo II. Se ahonda en tres rasgos que representan mejor la naturaleza femenina: interioridad y apertura, maternidad y cuidado de la vida, fortaleza y conciencia de la debilidad; a partir de los cuales se ofrecen algunas sugerencias y experiencias educativas. 
En conclusión, el genio femenino, signado por la entrega y la solidaridad, puede materializarse en estrategias específicas que favorecen la enseñanza de la ética y de las humanidades, para contribuir al desarrollo de una cultura en favor de la vida y de un más arraigado compromiso social con la persona y su dignidad.

Palabras clave: Mujer; Bioética; Cuidado de la Vida; Genio femenino; Educación.

\begin{abstract}
The urgent demand for a more incisive presence of women in political, social, and cultural life has been made from different perspectives and diverse motivations. However, the specific contribution that only women can make could be frustrated when women's constitutive characteristics are neglected. Due to this risk, the educative I field is an important scenario to comprehend the feminine essence and to permeate the teaching practices. This article is the result of research and teaching of bioethics inserted in the curriculum of Humanities and Nursing. The purpose is to delve into the qualities of the authentic feminine to show what extent they could enrich the university educational praxis. The theoretical references are mainly the anthropological reflections of Edith Stein and John Paul II about women. The three traits that best represent the feminine nature were developed: interiority and openness, motherhood and care for life, strength and awareness of weakness; from which we offer some suggestions and educational experiences.

In conclusion, the feminine genius, characterized by dedication and solidarity, can be evidenced in specific strategies that favor ethics and humanities teaching. It contributes to the development of a pro-life culture and a more deeply rooted social commitment to human dignity.
\end{abstract}

Keywords: Women; Bioethics; Care for life; Feminine genius; Education.

\title{
Introducción
}

El presente artículo parte de la preocupación docente por brindar una sólida formación ética a estudiantes universitarios, en quienes se constata, en los últimos años, una creciente influencia de lo que el Papa Juan Pablo II llamó "cultura de la muerte" (Velásquez Portilla et al., 2021; Brandalise et al., 2018; Huamani y Serruto, 2017; López-Luján et al., 2014).

Esta es cada vez más palpable en la sociedad actual, en donde, a través de sus instituciones, los medios de comunicación y la modificación de la legislación se favorece y promueve, como una expresión aparentemente "legítima" de la libertad individual, la eliminación de algunos seres humanos en situación de vulnerabilidad como los niños no nacidos, los ancianos y los enfermos. Así lo demuestran, entre otros, las leyes y movimientos pro-aborto, eutanasia y suicidio asistido.

Esta mentalidad, marcada por la deshumanización y la falta de solidaridad ante el más débil e indefenso, abarca actitudes y comportamientos contrarios al valor de la vida y al respeto por la dignidad de toda persona humana, es fruto de una distorsionada manera de ver la realidad del otro en cuanto ser humano sufriente y de la paulatina deformación de la 
conciencia moral que ha llevado a proclamar como "derechos" los que son auténticos delitos (Santa Cruz-Vera, 2017).

Como se lee en la Evangelium vitae:

La vida, que exigiría más acogida, amor y cuidado, es tenida por inútil, o considerada un peso insoportable $y$, por tanto, despreciada de muchos modos. Quien con su enfermedad, con su minusvalidez, o más simplemente con su misma presencia pone en discusión el bienestar y el estilo de vida de los más aventajados, tiende a ser visto como un enemigo del que hay que defenderse o a quien eliminar (Juan Pablo II, 1995, ก. 12).

Ante este panorama, la educación superior se presenta como una palestra idónea para generar una cultura en favor de la vida y del cuidado del otro. Con palabras del Papa Francisco, se puede afirmar que: "Las universidades, por su naturaleza, están llamadas a ser laboratorios de diálogo y de encuentro al servicio de la verdad, de la justicia y de la defensa de la dignidad humana en todos los niveles" (Francisco, 2018).

Un reto de la educación superior es ayudar a contrarrestar la cultura de la muerte y del descarte a través de una educación ética sensible a los grandes valores de la solidaridad, la fidelidad a la conciencia y la acogida del otro en razón de su humanidad. Es válido para toda comunidad académica el mensaje del Papa a la Universidad de Tokio:

Ningún estudiante [...] debería graduarse sin haber aprendido cómo elegir, responsable y libremente, lo que en conciencia sabe que es lo mejor. Que en cada situación, incluso en las más complejas, se interesen por lo que en su conducta es justo y humano, cabal y responsable, decididos defensores de los vulnerables (Francisco, 2019).

Un aporte imprescindible, en la línea de una formación humanística, y humanizante, dentro del medio universitario, puede venir, como lo sugiere Redondo (2020), de permear la tarea educativa de aquello que constituye lo auténtico femenino y que se manifiesta en la opción de poner en el centro de la educación "las cuestiones de sentido, la primacía de la persona y su dignidad, la ética, la solidaridad con la sociedad y especialmente los más débiles, la educación en las virtudes, el matrimonio y la familia" (p. 115).

La mujer, por el singular privilegio de alumbrar la vida, protegerla en su seno y hacerla crecer, desarrolla una peculiar sensibilidad hacia el otro; su misión y dignidad se ligan al amor que da y que recibe, a la acogida, la entrega y la solidaridad con la vida humana más indefensa. Esta íntima relación que la mujer guarda con la vida gesta en ella disposiciones 
adecuadas para ser madre y educadora. Es propio de la mujer humanizar, con su sola presencia, las relaciones sociales, enseñar a privilegiar el ser sobre el tener, la compasión sobre la utilidad, el amor sobre el poder.

De ahí que, para una adecuada formación bioética, parecen propicias aquellas iniciativas y estrategias educativas que se propongan impregnar con la riqueza de la feminidad la vida universitaria, con el fin de rescatar lo que es esencialmente humano y generar una cultura de la vida y del cuidado del otro. Una educación que sea capaz de tener en cuenta todas las dimensiones de la persona: no solo la intelectiva sino también la psicológico-afectiva, moral y espiritual.

En el marco de la antropología personalista, esta investigación tiene como objetivo profundizar en las cualidades de lo auténtico femenino e indagar y mostrar en qué medida dichas cualidades pueden enriquecer la praxis educativa universitaria, contribuir al desarrollo de una cultura en favor de la vida y de un más arraigado compromiso social con la persona y su dignidad. Para ello, en un primer momento se ahondará en las características que constituyen la esencia femenina, para presentar, en segunda instancia y a partir de las mismas, algunas sugerencias y reflexiones educativas en el ámbito de las humanidades en general y de la enfermería en particular. Se finalizará el escrito con algunas conclusiones.

\section{La esencia femenina: fundamentación antropológica del ser de la mujer}

En la reflexión acerca de la mujer, y dentro del movimiento feminista de principios del siglo XX, es la filósofa alemana Edith Stein (2001), quien aborda esta inquietud por encontrar el fundamento antropológico de la mujer, su "esencia". Sus trabajos nos acercan a lo que más tarde Juan Pablo II llamó el "genio femenino" y que consiste en aquello que específicamente caracteriza a la mujer por ser mujer. Se trata de rasgos -cualidades o capacidades- que por ser humanas no son exclusivos del sexo femenino, pero sí definen a la mujer porque en ella se dan de forma predominante, y en el varón de forma minoritaria y, a menudo, requiere un esfuerzo de desarrollo que la mujer realiza con más facilidad.

Por otro lado, es Juan Pablo II, y su propuesta de una antropología adecuada, quien ha penetrado en el núcleo mismo del "genio femenino", su vocación a la maternidad física y espiritual, en su misión y dignidad; intuiciones y desarrollos que han quedado plasmados en sus diversas intervenciones, catequesis, cartas y, principalmente, en la Exhortación apostólica Mulieris dignitatem.

Es a partir de estos autores y de sus reflexiones antropológicas sobre la mujer de donde se extraerá aquellos rasgos que representan mejor la naturaleza femenina, haciendo notar que 
cada uno de ellos es también compartido por el varón, puesto que hombre y mujer son dos versiones originales del ser humano único creado a imagen y semejanza de Dios.

\subsection{Interioridad $y$ apertura a la persona}

Una característica propia de la persona humana -varón y mujer- es la receptividad o apertura a la realidad. Esta capacidad de percibir, entender el mundo y explicarlo, aplicado a otro ser humano se traduce en la posibilidad de comprenderlo y acogerlo en la propia interioridad (Santa Cruz-Vera, 2012).

Stein, en su acercamiento a la naturaleza femenina, descubre que, si bien toda persona participa de una triple condición corporal, anímica y espiritual, en la mujer la vinculación entre el cuerpo y el alma es más estrecha que en el varón. La mujer "no sólo siente su cuerpo, sino que siente y piensa con su cuerpo" (Stubbermann, 2003, p. 160) y, por tanto, participa con toda su persona en lo que hace. La integración de cuerpo, mente y corazón es en ella más fuerte que en el varón y la dota de una más honda unidad interior, un conocimiento más intuitivo de la realidad personal.

Como escribirá Seifert: "Es precisamente [...] en esa coexistencia del querer con el pensar y el sentir, en donde se encuentra la «magia de lo femenino»"; es esta unidad interior lo que hace a la mujer tan atrayente (2008). Para el varón, por el contrario, es más fácil la "división", el dejar a un lado los sentimientos del corazón, para focalizar su atención en un trabajo concreto; para la mujer, el hecho de relegar a un segundo plano los ecos de su afectividad, es algo mucho más difícil, en orden, justamente a la unidad del alma femenina que está toda entera en todo lo que hace (Guerra, 2005; Sordo, 2015).

Como escribe Gallardo (2016), siguiendo a Edith Stein:

De todas las facultades humanas, la fuerza de la mujer reside en la vida de su corazón, mientras que la fuerza del varón estaría en el conocimiento y la actuación. Esto significa que la interioridad masculina es más intelectual, mientras que la femenina es más afectiva (p. 104).

Es el corazón lo que transforma el conocimiento en una realidad asimilada interiormente. Es la riqueza de la afectividad lo que dispone a la mujer para una atención a la persona del otro, tanto en su totalidad como en su particular necesidad existencial; es la cualidad de recibir al otro en su intimidad, lo que le brinda una singular capacidad de comprensión e identificación con la interioridad y las vivencias de la otra persona. 
Al predominar, en la mujer, la afectividad (Wojtyla, 2013), percibe la realidad más hondamente, apropiándosela dentro de sí y quedando implicada en ella. El don de la acogida que caracteriza a la mujer le viene de su receptividad activa, libremente querida, con la que ella se compromete desde su interior y con toda su persona: inteligencia, voluntad y afectos. En razón de ello, la mujer posee una especial capacidad para amar y acompañar, pues sabe compadecerse más fácilmente: entrar en sintonía con el sentir del otro, sus vivencias y experiencias más profundas.

Para Stein, la respuesta típicamente femenina es la afectiva -que no sentimental-: su corazón se deja conmover -"afectar"- más profundamente por los acontecimientos, los valores y las personas. He ahí la riqueza genuina de la mujer: un corazón que sabe acoger y abrirse a la realidad de manera profunda.

En síntesis, la esencia femenina está marcada por la unidad interior, es decir que su conocer, su vivir y su obrar, tienen origen en su intimidad. Todo ello se manifiesta, en una especial preocupación hacia lo personal-vital que dota a la mujer de una aptitud natural para cuidar y promover la vida en todas sus facetas (Stein, 2001).

\subsection{El cuidado de la vida: don de la entrega y la solidaridad}

Juan Pablo II, en sus Catequesis sobre el amor humano, recogidas en el libro "Hombre y mujer lo creó" (2000), nos habla del lenguaje o la hermenéutica del cuerpo. En el cuerpo humano sexuado, varón y mujer, se encuentra inscrito un mensaje antropológico de capital importancia: hombre y mujer no agotan por sí solos la humanidad, no están hechos para la soledad o el egoísmo, la competitividad o el individualismo, sino que su cuerpo sexuado es signo de su vocación al amor, este les recuerda su destino, los reenvía constantemente al otro, diferente y complementario al mismo tiempo, a quien están llamados a entregarse y acoger como un don. Apunta aquí la vocación a la comunión de las personas de la que el cuerpo es sacramento -signo visible-. Este ser para el otro, y con el otro, en que consiste la comunión personal, define a la persona y el camino de su plenitud.

Asimismo, en la mujer se encuentra un hecho irrefutable: su corporeidad, está signada por la maternidad. El cuerpo femenino "se presenta estructurado de tal manera que pueda generar vida y, por tanto, [la mujer] no puede tener conciencia de sí misma sin referencia a este dato que constituye íntimamente su identidad" (Di Nicola 2009, p. 73).

"Lo materno" no es una capacidad solo biológica de alumbrar la vida, sino una cualidad de orden antropológico-espiritual que la dispone a amar, acoger y cuidar incondicionalmente a 
todo ser humano; es un fenómeno de gran profundidad que caracteriza a la mujer, es el núcleo esencial del "genio femenino".

Como lo afirma Santa Cruz-Vera:

Esta vocación a ser madre, estructura toda la personalidad femenina -en sus dimensiones biológica, psíquica y espiritual- dotándola de una disponibilidad interior para acoger al otro, darle un lugar, protegerlo, ayudarle a su desarrollo y crecimiento, dedicarse a él en cuerpo y alma [...] (2012, p. 212).

La consecuencia antropológica fundamental de esta comunión particularísima entre la madre y el niño, nos la presenta Juan Pablo II (1988):

Este modo único de contacto con el nuevo hombre que se está formando crea a su vez una actitud hacia el hombre -no sólo hacia el propio hijo, sino hacia el hombre en general- que caracteriza profundamente toda la personalidad de la mujer (n. 18).

Por esta llamada singular a entrar en contacto con el misterio de la vida, la mujer es más sensible al valor de la persona, percibe más fuertemente el imperativo ético para proteger y cuidar al otro, aun a costa de la propia vida. En ese gestar y dar a luz, la mujer empeña no solo las energías de su cuerpo, sino toda su persona: inteligencia, voluntad y su rica sensibilidad.

En esta misma línea, Edith Stein descubre en la mujer una doble tarea que parte de un anhelo profundo presente en el corazón femenino: "(...) desplegar y madurar del mejor modo posible la humanidad que duerme en ella (...), y a la vez, incentivar y promover en los demás la maduración en orden de su plenitud" (citado en Stubbemann, 2003, p. 161), es decir, que la humanidad que le ha sido confiada, alcance su máxima realización.

La realización de la persona se encuentra, no solo en el orden de lo físico-corporal, sino, principalmente, en lo ético-espiritual, en el orden de los grandes valores de la vida: la compasión, la solidaridad, la justicia, el bien, la belleza y la verdad. De ahí la importancia de la mujer como "primera educadora". La vocación a ser madre y custodia de la vida se realiza, más que en el plano meramente biológico, en el antropológico-espiritual: el de las relaciones interpersonales fecundas, de la confianza y el amor. $Y$ es que solo el amor hace crecer porque solo el amor es digno de fe.

Esta tarea del cuidado del otro está en estrecha relación con la capacidad de intuir necesidades ajenas y de adaptarse a circunstancias o acontecimientos inesperados. La madre que, en el embarazo, se ha abierto a la nueva vida generada en su seno, y que ha 
experimentado los cambios de su cuerpo adaptándose a la vida que late dentro, sabe, mejor que el varón, hacer "sitio" a cada persona humana, aceptar y acoger al otro que se presenta, muchas veces, débil e indefenso, revestido solo con el valor de su humanidad. Con los "ojos" propios del corazón femenino es capaz de descubrir el valor de cada hombre y entregarse a él con generosidad. En la afirmación constante del otro, la mujer afirma su propia identidad.

\subsection{Fortaleza y consciencia de la debilidad}

En la mujer se constata una acentuada consciencia de la vulnerabilidad humana. Los ritmos propios de su fisiología, y del proceso del embarazo, enseñan a la mujer a sobrellevar los cambios hormonales -con sus repercusiones anímicas y físicas-, las incomodidades, incertidumbre, o sufrimiento que conllevan. Es frecuente, además, que muchas mujeres se enfrenten al dolor por la pérdida de un hijo que no llega a nacer, o al anhelo, no cumplido, de ser madres.

Todos estos límites, propios de la condición humana, son experimentados por la mujer desde dentro y la convierten, por ello, en un signo visible del gozo que puede encerrar el sufrimiento o la esperanza escondida en el dolor. En palabras de Jesús:

La mujer, cuando va a dar a luz, está triste, porque le ha llegado su hora, pero cuando ha dado a luz al niño, ya no se acuerda del aprieto por el gozo de que ha nacido un hombre al mundo (Jn 16, 21).

La mujer se convierte así en maestra de vida, en aquella que recuerda el sentido y fecundidad que pueden brotar del sufrimiento, a condición de aceptar con humildad la naturaleza de criaturas, de asumir con paciencia y fortaleza la propia fragilidad, de aprender a amar y a transformar en madurez personal las dificultades de la vida.

¿De dónde obtiene la mujer su singular fortaleza y capacidad de sacrificio? Juan Pablo II (1988) responde: "La fuerza moral de la mujer, su fuerza espiritual, se une a la conciencia de que Dios le confía de un modo especial el hombre" (n. 30). Se puede colegir, entonces, que esta fortaleza para sufrir por aquellos que ama, estar presente en el misterio de la vida que nace, en el lecho de la enfermedad y de la muerte, proceden de su capacidad para amar, que en ella se traducen en don y entrega incondicional al otro. 


\section{Experiencia y sugerencias educativas en el ámbito de las Humanidades}

A partir de las características esenciales del genio femenino, se presentarán algunas experiencias educativas, en el ámbito de la enseñanza de la ética y la antropología filosófica, con las que se pretende promover en los estudiantes una especial sintonía con la dignidad de la persona y una cultura en favor de la vida.

\subsection{Cultivo de la interioridad}

Cada persona tiene la tarea de profundizar en el conocimiento de sí mismo, que en otras palabras significa conocer el mundo interior al cual solo él puede acceder, a partir de allí se alcanza mayor comprensión de la riqueza personal, para después, compartirla con los otros de manera más consciente. Es por ello que, haciendo eco de la capacidad femenina para conocerse a sí misma, conocer al otro e involucrarse con las vivencias existenciales de la persona concreta, se pueden proponer ejercicios didácticos que redunden en el cultivo y desarrollo de la interioridad.

Metáfora del árbol: una herramienta útil es el uso de metáforas para aplicar los contenidos de la clase en la propia vida. Por ejemplo, se puede recurrir a la figura del árbol con su estructura básica de raíces, tronco y frutos para representar algunos elementos constitutivos de la persona y de su dinamismo moral, tales como: opción fundamental, temperamento, talentos, valores (raíces); hábitos y actitudes adquiridas (tronco); virtudes y vicios (frutos) (figura 1 ).

Es recomendable que los jóvenes apliquen la creatividad y el trabajo manual en el desarrollo del esquema; los estudiantes pueden diseñarlo a su gusto, puesto que el tiempo que dedican a la construcción favorece también la reflexión. Otra adición que enriquece el ejercicio es validar y completar la información consignada con la que les brinda una persona que los conoce bien. Finalmente, y este es un elemento clave, deben elegir uno de sus malos hábitos, el que más los afecte a ellos y a su familia, y establecer una acción para corregirlo. En este punto el docente puede brindar orientación para que la acción sea concreta y guarde relación directa con lo que se desea cambiar. 


\section{Figura 1}

Ilustración metáfora del árbol

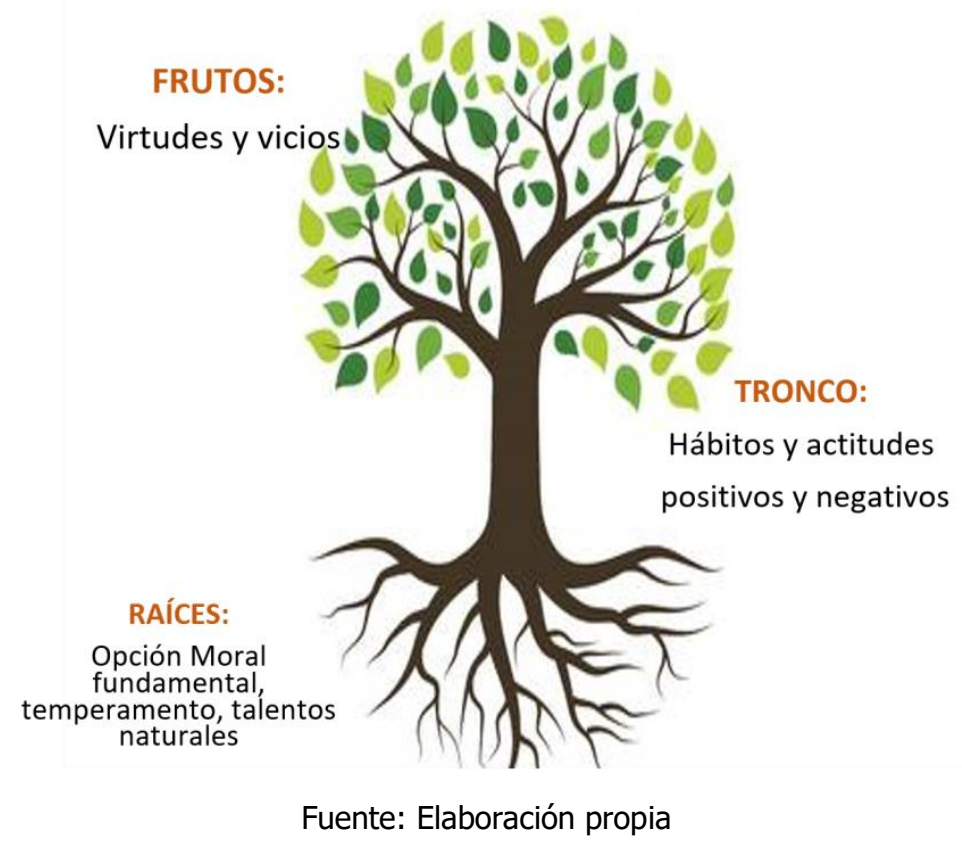

Cultivo de las virtudes: con respecto a la enseñanza de la virtudes, los educadores pueden encontrar cierta dificultad para conseguir que los jóvenes universitarios se interesen y comprometan vitalmente con el desarrollo de virtudes, no porque no adviertan su importancia para una vida recta, sino porque no se logra vislumbrar qué camino recorrer para alcanzar, no todas las virtudes de manera indistinta, sino aquella que es el "reto" de la vida personal (virtud reto), en orden a una sana convivencia y consecución de la felicidad.

Estas dificultades pueden ser superadas con la propuesta educativa de Liderazgo Virtuoso (Havard, 2017) puesto que consigue atraer el interés de los jóvenes al ofrecerles "herramientas" concretas para practicar las virtudes, según el temperamento de cada uno, con miras a cumplir su misión de vida. Además, ofrece una estructura temática que se engrana bien con los contenidos de la ética profesional, por ser los pilares formativos de este sistema el autoconocimiento y el desarrollo del carácter.

Toda persona llamada a ser líder primero debe conocer cuáles son las fortalezas y los desafíos del temperamento, cuáles son los talentos que se poseen, cómo perfeccionarlos y multiplicarlos para servir con ellos a los demás. En segundo lugar, debe empeñarse en forjar una personalidad honda, recia y atrayente, para ello es indispensable que ennoblezca su carácter a través de la virtud. 
En la experiencia docente, se ha constatado que este tema genera un impacto positivo en la vida personal de los estudiantes porque adquieren mayor comprensión de sí mismos y de los demás, y con ello, una actitud más favorable hacia la acogida del otro tal cual es. También experimentan gran satisfacción al observar mejoras en sus relaciones, en su rendimiento, y en sus hábitos después de empeñarse algunas semanas en la práctica de su virtud reto. Igualmente, en el ámbito laboral, algunos estudiantes, aunque son muy jóvenes, lideran en sus empresas equipos de trabajo y frecuentemente manifiestan que este tema les ha ayudado a mejorar sus competencias de liderazgo. Por esta razón, el liderazgo virtuoso permite a los jóvenes esculpirse una personalidad no solo atractiva sino enérgica y comprometida para generar una contribución real en la sociedad.

\subsection{Sensibilidad y cuidado de la vida}

La enseñanza de la bioética permite integrar diversas estrategias para lograr una comprensión más profunda de los contenidos y desarrollar un sentido de sensibilidad por el cuidado de la vida. A continuación, se presentan algunas sugerencias para dicho fin.

Voluntariado: es una eficaz estrategia alternativa a los métodos de enseñanza tradicionales, genera un aprendizaje significativo en los jóvenes porque entran en contacto directo con otras personas que los enriquecen a través sus historias de vida, además porque salen de ellos mismos, entregan sus talentos y desarrollan competencias que fortalecen su vida personal, profesional y espiritual (Nossa-Ramos, 2018; Santa Cruz-Vera y Bolívar, 2021a; 2021b). Para lograr estos resultados la clave está en ofrecer orientación, pero dejar que los jóvenes asuman el liderazgo durante la preparación y el desarrollo de la actividad de proyección social.

Inicio de la vida: por otra parte, una buena estrategia para fomentar la cultura de la vida es hacer especial énfasis en las etapas del desarrollo prenatal. A modo de introducción, es pertinente disponer a los estudiantes con una actividad que los involucre a ellos, personalmente, con el tema. Consiste en pedirles que recolecten información sobre su etapa prenatal y primeros años de vida por medio de algunas preguntas sugeridas por el profesor (figura 2). 


\section{Figura 2}

Ejercicio información prenatal

\section{Mis Primenos días}

¿Qué sabes acerca de tus primeros días de vida -cuando estabas en el ivientre de mamá!-?

\section{1. ¿Cómo fue el embarazo?}

Tu comportamiento en el embarazo: inquieto, tranquilo, etc.

2. Miedos o dificultades familiares. ¿Cómo las superaron?

3. ¿Cuándo y cómo escogieron tu nombre?

4. ¿Cómo prepararon tu llegada?

5. ¿Fue un parto difícil?

6. Recuerdos o anécdotas de tus primeros meses después del nacimiento

Fuente: Elaboración propia

Este ejercicio representa para muchos jóvenes la posibilidad de acceder a información importante sobre su vida, como por ejemplo la manera en que sus padres superaron las dificultades alrededor de su nacimiento: algunos comparten que sus padres pensaron en abortarlos, pero decidieron no hacerlo y ahora están muy orgullosos de sus hijos; otros dicen que llegaron en medio de penurias económicas y resaltan la audacia de sus padres para fundar una familia a pesar de las dificultades; otro indica cierto desconcierto por parte de los padres ante la noticia de su nacimiento porque ya era una familia numerosa, luego, en su infancia, tuvo problemas de aprendizaje y justamente sus hermanos fueron los mejores terapeutas que le ayudaron a superarlos; otros pasaron por complicaciones graves en la gestación o en el parto, y también gracias a la fe y al amor de sus padres y familiares salieron adelante; y la mayoría manifiestan que sus padres sintieron gran alegría por su vida.

En fin, son muchas las experiencias que los ayudan a asombrarse ante la vida, a redescubrir su valor, su belleza y, todo ello, los dispone para abordar el tema del inicio de la vida con una postura más positiva y reflexiva.

Desarrollo prenatal: Hoy en día es necesario informar acerca de las etapas iniciales de la vida humana, porque hay desconocimiento alrededor de ello, especialmente en la juventud. 
Además, a los jóvenes les agrada recibir datos desde la ciencia porque consideran que es información objetiva y les ayuda a tener criterios sólidos. Para trabajar este tema se pueden sugerir preguntas con el objetivo de orientar una consulta rápida y suscitar interés (figura 3). Después, el docente hace la explicación y, como actividad evaluativa, los jóvenes presentan un esquema gráfico o infografía con las etapas del desarrollo prenatal. Esta actividad, de consulta y organización de la información, les resulta atractiva y favorece su proceso de aprendizaje.

\section{Figura 3}

Preguntas desarrollo prenatal

\section{Desannollo Prenatal}

1. ¿Cuándo se da la implantación?

2. ¿Cuáles son los primeros órganos que se forman?

3. ¿Cuándo inician las contracciones en el músculo del corazón?

4. ¿Cuándo está completo el sistema nervioso?

5. ¿En qué semana se completa el esqueleto?

6. ¿Cuándo puede sentir dolor?

7. ¿A los cuántos meses, da patadas y puede llorar?

Fuente: Elaboración propia

Síndrome postaborto: A veces es una buena estrategia no abordar directamente el tema del aborto, sino profundizar más bien en el síndrome postaborto -que hace referencia a la sintomatología que se desencadena después de un aborto- y recurrir a la voz de los expertos por medio de artículos científicos, conferencias e, incluso, alguien que lo haya padecido; estas personas están más disponibles de lo que se piensa para compartir su experiencia y es posible localizarlos en fundaciones provida. Los testimonios suelen tener buena acogida y, si no se cuenta con uno directo, se puede seleccionar material adecuado disponible en la red.

Este tema es importante porque revela a los jóvenes una cara oculta del aborto: el sufrimiento de hombres y mujeres que han perdido un hijo (Melina y Anderson, 2010) y, a raíz de ello, aflora la conciencia de que, entre ellos, hay jóvenes que lo padecen y que es necesario adoptar una postura más delicada cuando se opina sobre el tema. En el ambiente universitario es frecuente encontrar estudiantes que sufren o han pasado por este síndrome y toman la iniciativa para compartirlo a solas con el docente; en general, la mayoría 
confirma el sufrimiento y arrepentimiento experimentado, y la necesidad de compartirlo con alguien que los comprenda.

\subsection{Fortaleza y consciencia de la debilidad}

En la enseñanza de la bioética es bueno no perder de vista el método triangular propuesto por Sgreccia (2003) que consiste en confrontar el dato médico, el componente antropológico y la valoración ética para hacer un análisis global del problema en cuestión. En este sentido, es pertinente el enfoque de totalidad que la esencia femenina ofrece porque, en la práctica, los jóvenes necesitan una explicación metódica que aborde diferentes caras de la realidad y les ayude a ampliar su visión y comprensión del tema.

En el caso concreto del final de la vida, se sugiere hacer énfasis en el componente antropológico desarrollando temas como el sentido del sufrimiento, la muerte y la vulnerabilidad (Santa Cruz-Vera y Bolívar, 2021b), esto evita el reduccionismo en el análisis y es una manera más humana de abordarlo.

Actividades de contextualización: En algunas ocasiones, los jóvenes presentan actitudes de rechazo ante ciertos temas porque los docentes no generan siempre una atmósfera apropiada que facilite la acogida de lo que se transmite. Por ejemplo, en el tema del final de la vida es importante crear contexto con lecturas que susciten la reflexión en torno a elementos antropológicos y destinar un momento para compartir las impresiones en grupos de discusión.

También puede ser útil leer un libro a lo largo del curso, que cuente una historia de vida real y deje enseñanzas importantes con relación al sufrimiento, la muerte y las virtudes. Aquí se recomienda el libro "Sobrevivir para contarlo" de Inmaculée Illibagiza, una joven ruandesa que, junto con su familia, afrontó de manera admirable el holocausto desatado en su país en 1997. Es un libro escrito con un estilo ágil que atrae a los jóvenes y con adecuada sensibilidad moral, que favorece su formación. Por ello, son muchas las enseñanzas que ellos recogen de esta historia, pero en especial señalan la importancia de la fortaleza, la fe y el perdón en medio de grandísimos sufrimientos.

Proyectos integradores: En el ambiente universitario es viable crear espacios de estudio de la bioética, que integren docencia, extensión e investigación. Un tema que vale la pena profundizar, para contrarrestar el discurso pro-eutanasia y fomentar la cultura de la vida, es 
el de los cuidados paliativos. Por medio de ejercicios investigativos y de experiencias con personal médico y pacientes en hospices y hospitales, los jóvenes pueden descubrir y practicar la ética del cuidado, no solo con los pacientes terminales, sino también en todos los ámbitos de la vida; la clave es aprenden a cuidar y acompañar al otro en su sufrimiento.

\section{Experiencias bioéticas en el ámbito de la Enfermería}

Las experiencias educativas en enfermería, tal vez por ser esta profesión fruto de la tarea de muchas mujeres a lo largo de la historia, se ven empapadas del ejercicio y aplicación del genio femenino dado que el compromiso del profesional de enfermería es defender la vida en sí misma, reconociéndole a cada persona su dignidad, intimidad y autonomía (Alvarado García, 2004).

Se cita a continuación un caso que ayuda a iluminar la reflexión que se está desarrollando. Al retomar las prácticas de enfermería, un grupo de estudiantes entró en contacto con un paciente de 80 años, quien tenía antecedentes de Alzheimer. Estaba hospitalizado en la institución por una sepsis secundaria a dos grandes heridas, dado que vivía con su esposa, también enferma, y era cuidado por su hija, quien al contagiarse de COVID no pudo ayudarle en la movilización sufriendo entonces el detrimento de su situación clínica.

Durante la hospitalización era atendido por su hija y una cuidadora, quienes se observaban claramente agotadas por todo el proceso, su hija además presentaba conflictos familiares, puesto que la culpaban del deterioro de su padre. Por todo lo anterior, con las estudiantes, nos dedicamos a ayudar en el cuidado del paciente para favorecer su recuperación. Con el paso de los días dejó de comer y presentó episodios de apnea respiratoria, ante esto, el personal médico de urgencias, decidió no intervenirlo en dicha situación clínica. Reposicionamos al paciente para que pudiera oxigenarse mejor y, no sin desazón, terminamos ese turno de práctica.

Al siguiente turno encontramos que el médico tratante había implementado algunas medidas que podrían recuperar al paciente, y con todo empeño nos dedicamos a ayudarle. El resultado: al cabo de unas semanas el paciente fue dado de alta, sin apneas respiratorias, ingiriendo alimentos por la vía oral y con cuidados domiciliarios para sus heridas. Pudo reencontrarse con su esposa, quien estaba agobiada al no poder visitarlo, logró compartir la navidad con su familia y pasar sus últimos días con quienes más amaba.

Durante el proceso, una estudiante, por ejemplo, estaba indignada por la atención brindada por el personal de urgencias, pues recientemente había perdido a su abuela por la misma 
enfermedad, pero en condiciones más humanas. Otras cuestionaban la necesidad de avanzar en las medidas terapéuticas y la situación bioética al final de la vida se puso en discusión. Llegamos al acuerdo de que, si bien el paciente no se recuperaría, si había dos posibilidades para su atención: una sin la terapia correspondiente dejando que sus enfermedades avanzaran y muriera en el hospital, la segunda con una atención que le permitiera reponerse lo suficiente para regresar a su hogar y pasar sus últimos días en compañía de sus seres amados, que fue finalmente la que sucedió.

A raíz de esta experiencia, se logró enseñar a las estudiantes las virtudes necesarias para el ejercicio consciente de su tarea: la empatía, la comprensión, la acogida del otro, en últimas, una mirada humana más amplia en equilibrio con la mirada objetiva. En este caso, se evidencia cómo el genio femenino aporta su capacidad de escucha, su inteligencia, habilidad organizativa y de coordinación para la solución de problemas (López-Ibor Aliño, 2020). Asimismo, se deriva un doble reto para los profesores: cultivar estos rasgos en sí mismos y transmitirlos a sus estudiantes.

Se demuestra, también, cómo la familia y el paciente depositan su confianza y dan por sentado que, las personas encargadas de la atención en salud cuentan con la formación necesaria para asumir su cuidado (López-Ibor Aliño, 2020). En este momento el estudiante se reconoce como un ser vulnerable y falible, que cuenta con la disposición para cuidar al otro, pero que carece de la experiencia, y por ende es sujeto de temores y angustias, es frecuente escuchar comentarios del tipo: "¿y si me equivoco?, ¿si no soy capaz?", es en este momento donde el ejercicio del docente consiste no sólo en dar seguridad y orientación al estudiante, sino en lanzarlo a superar su ansiedad, recordarle los principios técnicos y científicos que guían su acción y acompañarlo, como una madre a sus hijos, en sus primeros pasos. Esto contribuye a la adquisición de habilidades en el razonamiento ético-clínico y de experticia práctica (Alvarado García, 2004).

\section{Conclusiones}

El objetivo de este trabajo era ahondar en las cualidades esenciales del genio femenino con el fin de presentar algunas sugerencias educativas que enriquezcan el proceso de enseñanza-aprendizaje de la bioética. Como resultado de estas indagaciones se concluye que:

- El núcleo de la esencia femenina radica en capacidad ontológica de ser madre. De ella se deriva su disposición para acoger la vida, hacerla crecer y acompañarla hacia su pleno desarrollo. Esta actitud se expresa en un don especial para la entrega y la 
solidaridad, no solo hacia su propio hijo, sino hacia cada ser humano. A causa de esta vocación a la maternidad, la mujer entra más fácilmente en sintonía con el sentir del otro y sus vivencias más íntimas. Por último, la conciencia de ser custodia de la vida la reviste de una especial fortaleza para el sacrificio por el bien de la persona humana concreta.

- Un aporte concreto del genio femenino en la enseñanza de la bioética es el enfoque de totalidad tan pertinente para el análisis de los principales problemas de esta disciplina. Además, ante los eufemismos y manipulación ideológica de la "cultura de la muerte", tan presente en la sociedad actual, los jóvenes necesitan que se les ayude a trascender hacia una reflexión antropológica rigurosa. Esto demanda de los docentes una preparación ético-científica adecuada, junto con la aplicación de estrategias que permitan la positiva recepción de la verdad sobre el ser humano y sus exigencias morales.

- Por otro lado, la formación en el área de la salud precisa de la ética del cuidado que implica la capacidad de responder, con una más alta calidad humana, no solo a la dignidad de la persona sino a la necesidad concreta de cada paciente y de sus familias. Lo anterior redundaría en una mayor competencia profesional y, a largo plazo, en la mejora de los servicios de salud ofrecidos.

- Finalmente, el esfuerzo por impregnar la formación universitaria de las cualidades propias de lo auténtico femenino, contribuirá a fomentar, junto con unas relaciones interpersonales más fecundas, la cultura en favor de la vida.

\section{Referencias}

Alvarado García, A. (2004). La Ética del Cuidado. Aquichan, 4(4), 30-39.

Brandalise, V. B., Remor, A. P., Carvalho, D. D., y Bonamigo, E. L. (2018). Suicidio asistido y eutanasia en la perspectiva de profesionales y estudiantes de un hospital universitario. Revista Bioética, 26, 217-227. https://doi.org/10.1590/1983-80422018262242

Di Nicola, G.P. (2009). Yo masculino y Yo femenino. Ponencia presentada a las $V$ Jornadas de la Asociación Española de Personalismo, "Del qué al quién", Universidad CEU San Pablo, Madrid 2009. En: Fundamentos de la persona. Pp. 67-76. https://bit.ly/3pE7Q4m

Francisco (14 abril 2018). Discurso del Santo Padre Francisco a una delegación de la Villanova University de Filadelfia. https://bit.ly/31K5MiI

Francisco (26 de noviembre de 2019). Discurso del Santo Padre Francisco en su visita a la Universidad de Tokio. https://bit.ly/33ihfHn 
Gallardo, S. (2016). La interioridad de la mujer y santa Teresa de Jesús. En: Jiménez, L. (dir.). Educación de la interioridad tras las huellas teresianas. Fundación Universitaria Española. Pp. 99-109.

Guerra, R. (2005). Identidad Femenina y Humanización del Mundo. Revista Panamericana de Pedagogía, 7, 101-136

Havard, A. (2017). Liderazgo virtuoso. Palabra.

Huamani, J. C., y Serruto, A. (2017). Actitud hacia al aborto en estudiantes universitarios. Revista De Investigación En Psicología, 20(2), 363-375. https://doi.org/10.15381/rinvp.v20i2.14046

Juan Pablo II. (1988). Mulieris dignitatem. https://bit.ly/3oBWRZN

Juan Pablo II. (1995). Evangelium vitae. https://bit.ly/3IKP2PO

Juan Pablo II. (2000). Hombre y mujer lo creó. Ediciones Cristiandad.

López-Ibor Aliño, B. (2020). Bioética clínica: la mujer ante los retos de la bioética clínica actual. Apuntes de Bioética, 3(2), 39-47. https://doi.org/10.35383/apuntes.v3i2.497

López-Luján, D., Pou, M. T., y de la Cruz, L. (2014). Conocimiento, actitud y práctica de bioética en residentes médicos de Santiago de los Caballeros. Cuaderno De Pedagogía Universitaria, 10 (20), 39-45. https://doi.org/10.29197/cpu.v10i20.192

Melina, L. y Anderson, C.A. (2010). Aceite en las heridas. Análisis y respuestas a los dramas de aborto y del divorcio. Palabra.

Nossa-Ramos, D. (2018). Voluntariado universitario. Un espacio concreto para poner en práctica la vocación de servicio. En Garzón, E. (Comp.), Humanismo y mundo actual (25-38). Universidad Católica de Colombia. https://bit.ly/3EyJ2AV

Redondo, M. L. (2020). ¿Feminizar la Universidad? Una apuesta por la complementariedad. Apuntes De Bioética, 3(2), 105-120. https://doi.org/10.35383/apuntes.v3i2.476

Santa Cruz-Vera (2012). Lo auténtico femenino y la elevación de lo humano. Reflexiones a partir del pensamiento de Juan Pablo II. En: Cuadernos de pensamiento. Fundación Universitaria Española. Pp. 203-220.

Santa Cruz-Vera, D. J. (2017). Universidad Católica y cultura de la vida. En: Filosofía y personalismo en un mundo en crisis. Universidad Católica de Colombia. https://bit.ly/3DBtfQE

Santa Cruz-Vera, D. J., y Bolívar, M. (2021a).El voluntariado universitario al servicio de la Misión Institucional de la Universidad Católica de Colombia. En Santa Cruz-Vera, D. (Comp.), Reflexiones en torno a un proyecto educativo, (pp. 195-214). Universidad Católica de Colombia.

Santa Cruz-Vera, D. J., y Bolívar, M. (2021b). Sentido de la vida y del sufrimiento.: Una tarea personal. Apuntes de Bioética, 4(1), 5-22. https://doi.org/10.35383/apuntes.v4i1.624 
Seifert, J. (2008). Defender a la mujer del feminismo. La verdad ofende. https://bit.ly/3lrpcka

Sgreccia, E. (2003). Manuale di bioética. Vita e Pensiero.

Sordo, P. (2015). iViva la diferencia! (y el complemento también). Planeta.

Stein, E. (2001). La mujer (3. ${ }^{a}$ ed.). Biblioteca Palabra.

Stubbemann, C. M. (2003). La mujer en Edith Stein: antropología y espiritualidad. Imprenta Santos.

Velásquez Portilla M, Espejo Saavedra A, Navarrete Ospina F, Robledo Gómez S, Salazar Jaramillo M, Baquero García P, Buesaquillo C, Arango L, Burbano L, Andrade L, Calvache JA, De Vries E. (2021). Conocimientos acerca de la eutanasia en estudiantes universitarios en dos instituciones de educación superior en Colombia. Universitas Medica. 2021; 62(1). https://doi.org/10.11144/Javeriana.umed62-1.euta

Wojtyla, K. (2013). Amor y responsabilidad (9. a ed.). Palabra.

\section{Cómo citar este trabajo}

Santa Cruz Vera, D. J., Bolívar Ramírez, M., Tobón Gallego, L. I., \& Ortiz Núñez, S. (2021). Mujer y bioética. Experiencias y sugerencias educativas. Apuntes De Bioética, 4(2), 5-23.

https://doi.org/10.35383/apuntes.v4i2.679

\section{Financiación}

El presente artículo no cuenta con financiación específica de agencias de financiamiento en los sectores público o privado para su desarrollo y/o publicación.

\section{Conflicto de interés}

Los autores del artículo declaran no tener ningún conflicto de intereses en su realización.

(c) Los autores. Este artículo es publicado por la Revista Apuntes de Bioética del Instituto de Bioética, Universidad Católica Santo Toribio de Mogrovejo. que la obra original sea debidamente citada. 